\title{
Effects of Metamaterial Loading on Miniaturization of Loop and Open Loop Microstrip Filters
}

\author{
Saughar Jarchi \\ Faculty of Technical and Engineering, \\ Imam Khomeini International \\ University (IKIU), \\ Qazvin, Iran \\ s.jarchi@eng.ikiu.ac.ir
}

\author{
Mohsen Khalily \\ Institute for Communication Systems, \\ Home of the $5 G$ Innovation Center, \\ University of Surrey, \\ Guilford, UK \\ m.khalily@surrey.ac.uk
}

\author{
Rahim Tafazolli \\ Institute for Communication Systems, \\ Home of the $5 G$ Innovation Center, \\ University of Surrey, \\ Guilford, UK \\ r.tafazolli@surrey.ac.uk
}

\begin{abstract}
In this paper, metamaterial loading on loop and open loop microstrip filters is investigated where both rectangular loop and open loop structures are considered. Spiral resonators are loaded on the four sides of the square loop and result in higher size reduction compared to the conventional split ring resonators with identical structural parameters. It is shown that, for both proposed filters, metamaterial loading provides size reduction, due to possessing lower resonant frequency of spiral resonators. The structures are analytically investigated through the transmission matrix method. In the designed rectangular loop filters, there are two nulls on both sides of the pass-band, which provide high out-of-band rejection and is preserved in the corresponding miniaturized metamaterial loaded structures. However open loop resonators provide lower resonant frequencies or more compact size filters. The proposed filter is fabricated and tested and measured results are in good agreement with simulation ones.
\end{abstract}

Keywords—Loop filter, Open-loop filter, Metamaterial, Spiral Metamaterial, Miniaturization

\section{INTRODUCTION}

Wireless communication demand compact, low profile, and easy fabrication devices which necessitated the use of microstrip structures. Microstrip filters are inevitable parts of communication systems. Various filter structures are proposed and designed in literature.

Loop and open loop structures are of basic configurations to provide compact narrow band filters, and many researches have been performed in literature to understand and improve these filters' performance. Microstrip loop resonators to provide bandpass filtering properties are investigated in [1-3]. Compact designs are proposed in [4], [5]. A circuit model for coupling to ring resonators is proposed in [6]. With cascading multiple resonators, high rejection band property is achieved in [7]. An analytical method is developed in [8]. A method to control attenuation poles is proposed in [9].

Open loop resonators, a modification of loop resonators with lower resonant frequency, are investigated theoretically and experimentally in [10-12]. An open loop filter with hexagonal geometry is designed in [13]. A method to tune resonant frequency is discussed in [14].

Various designs in addition to loop and open loop resonators are proposed in literature. Compact microstrip filters are designed and fabricated in [15-22]. Most of the mentioned filters are designed for a specific frequency band and can't be extended for other frequency bands.

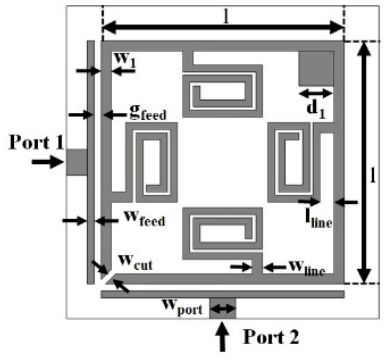

(a)

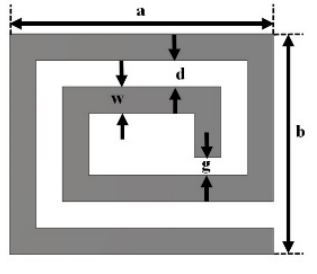

(b)
Fig. 1. (a) SP loaded loop resonator, (b) dimensions of the SP.

In this letter, compact bandpass filters based on rectangular loop and open-loop resonators loaded by metamaterial (MTM) cells, on continuous ground plane, are proposed and analytically investigated. Generalization of the filters for other frequency bands is straight forward and can be performed with scaling the loop and MTM cells and adjusting the dimensions for best performance. Changing the MTM cells' dimensions provides resonant frequency adjustment, which in this paper is determined for the lowest resonant frequency. Section II describes the structure geometry and provides analytical expressions. In Section III, designs based on the proposed structure are investigated. Measurement results are provided in Section IV. Conclusion remarks are in Section V.

\section{GEOMETRY OF THE PROPOSED STRUCTURE}

The basic resonator structure of this paper, rectangular loop resonator loaded by MTM cells, is shown in Fig. 1. The rectangular loop resonator should include some kind of perturbation to exhibit mode separation needed for bandpass filtering response, which is the square on top right corner of the loop with dimensions $d_{1}$. Open loop resonator is a capacitively loaded loop resonator with a narrow gap resembling the capacitance, shown by dimension $w_{c u t}$ in Fig. 1(a). The application of perturbation is not necessary for open loop resonators. Through loading the loop resonator by a capacitor, a lower resonance frequency would have resulted. These kinds of resonators are basically fed through the capacitive coupling, as shown in Fig. 1 with the shown dimensions.

\section{A. The proposed metamaterial resonator}

In this paper, all the four arms of the loop and open loop resonators are loaded by MTM cells, which have electrical 


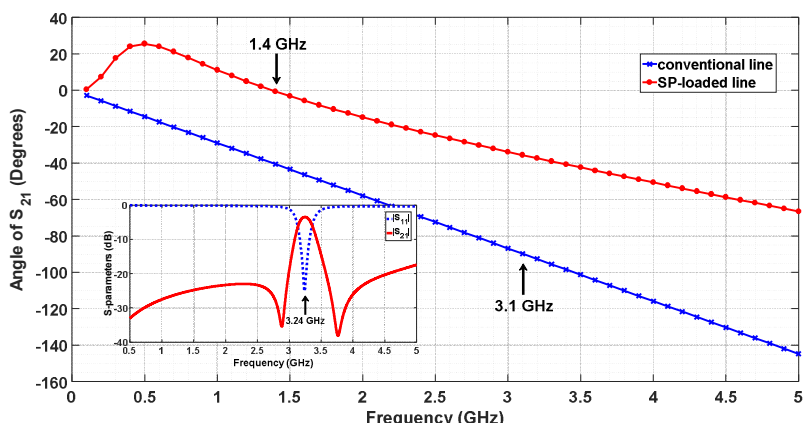

Fig. 2. Angle of $\mathrm{S}_{21}$ for conventional and SP-loaded $14 \mathrm{~mm}$ microstrip lines.

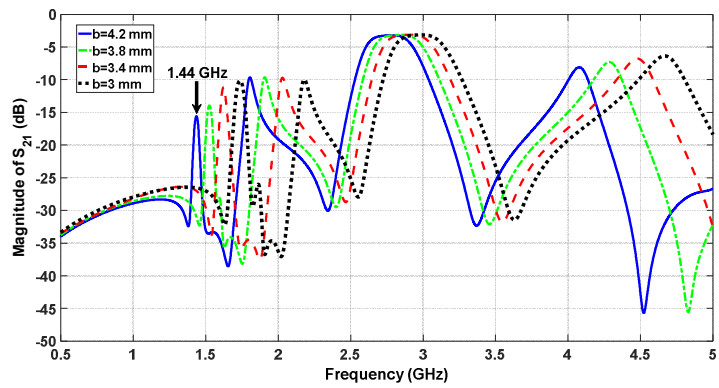

Fig. 3. Transmission coefficient of SP loaded loop resonator (dimensions: $\mathrm{W}_{\text {line }}=0.4 \mathrm{~mm}, \mathrm{l}_{\text {line }}=0.1 \mathrm{~mm}, \mathrm{w}_{1}=0.4 \mathrm{~mm}, \mathrm{~d}_{1}=3 \mathrm{~mm}, \mathrm{w}_{\mathrm{f}}=0.4 \mathrm{~mm}, \mathrm{~g}_{\mathrm{f}}=0.1$ $\mathrm{mm}, \mathrm{a}=4.2 \mathrm{~mm}, \mathrm{~d}=0.1 \mathrm{~mm}, \mathrm{w}=0.2 \mathrm{~mm}, \mathrm{~g}=0.2 \mathrm{~mm})$.

contact with the loop arms. MTMs are promising structures to enhance wireless devices' performance [23], [24]. Two kinds of MTM cells, split ring resonator (SRR) and spiral resonator (SP) may be considered. SPs represent higher equivalent capacitance and inductance compared to SRR cells and are approximately half the size of SRRs with the same resonant frequency. Therefore, to design the most compact filter, SP resonators are selected. The proposed loop resonator loaded by the two turn SP cells, as well as the corresponding dimensions, is illustrated in Fig. 1.

\section{B. Analytical Investigation}

To analytically investigate the effect of metamaterial loading, the ABCD matrix is used. Each arm of the loop resonator can be modeled through two transmission lines, with electrical length $\theta=\beta l$, loaded by impedance $\mathrm{Z}$ at the middle point. The impedance $Z$ represents the equivalent impedance of the metamaterial cell at the working frequency, parallel combination of the equivalent capacitance and inductance of the MTM cell, which is coupled to the ground plane through capacitance $C$. The coupling capacitance, $C$, is approximated as the capacitance between a unit length strip of width $\mathrm{w}$ and the ground plane and equals to:

$$
C=\epsilon_{0} \epsilon_{r} w / h s u b
$$

For simplicity, conduction and dielectric losses are ignored, which have no significant effect on the resonant frequency. For SP, the equivalent capacitance and inductance are calculated as the method of [25], [26] :

$$
\begin{aligned}
L^{S P} & =\frac{\mu_{0}}{2} l_{\text {ave }}^{S R}\left[\ln \left(\frac{l_{\text {ave }}^{S R}}{2 w}\right)+\frac{1}{2}\right] \\
C^{S R} & =\frac{l}{4(w+s)} \frac{N^{2}}{N^{2}+1}\left[l(N-1)-\frac{N^{2}-1}{2}(w+s)\right] C_{0},
\end{aligned}
$$

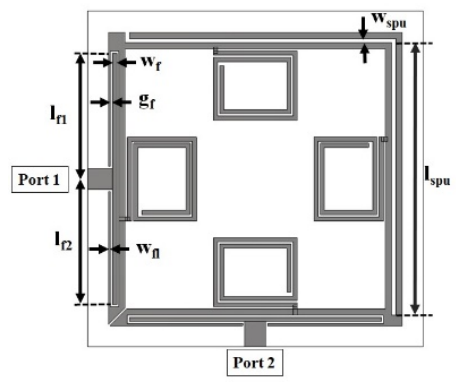

Fig. 4. Structure of the final filter.

$$
\begin{aligned}
& l_{\text {ave }}^{S R}=4 l-\left[2(N+1)-\frac{3}{N}\right](s+w) \\
& C_{0}=\epsilon_{0} \epsilon_{r}^{S u b}\left(\epsilon_{r}, h, w, s\right) \frac{K\left(\sqrt{1-\kappa^{2}}\right)}{K(\kappa)} \\
& \kappa=\frac{s}{s+2 w} \\
& \epsilon_{r}^{\text {sub }}\left(\epsilon_{r}, h, w, s\right)=1+\frac{2}{\pi} \tan ^{-1}\left[\frac{h}{2 \pi(w+s)}\right]\left(\epsilon_{r}-1\right) \\
& ,[0<(h / w),(h / s)<12 \pi]
\end{aligned}
$$

Here, $K$ is the elliptic integral; $N$, number of turns; 1 , side length of the external turn; w, width of the strips; and s, separation between two adjacent turns.

\section{SIMULATION RESULTS}

The proposed structures are designed on an FR4 substrate with a dielectric constant of 4.3 and thickness of $1 \mathrm{~mm}$, for low cost and easy availability. To investigate the effects of metamaterial loading on the loop filter, some analytical calculations are performed. First, an individual arm of the filter, with a length of $14 \mathrm{~mm}$, and a width of $0.4 \mathrm{~mm}$ is analyzed, and $\mathrm{S}_{21}$ is calculated and plotted in Fig. 2. It is observed that, at frequency of around $3.1 \mathrm{GHz}$, angle of $\mathrm{S}_{21}$ equals $-\pi / 2$, and therefore loop resonance occurs.

Then, the SP-loaded resonator is analyzed and the angle of $\mathrm{S}_{21}$ is calculated. The $\mathrm{SP}$ resonator dimensions are: $\mathrm{N}=2, \mathrm{w}$ $=0.2 \mathrm{~mm}, \mathrm{~d}=0.1 \mathrm{~mm}, \mathrm{a}=\mathrm{b}=3.6 \mathrm{~mm}$. The result is also depicted in Fig. 2. Metamaterial behavior, including a positive phase, is clearly seen. It is also observed that for SPloaded line at around $1.4 \mathrm{GHz}$ the angle of $\mathrm{S}_{21}$ is 0 . Therefore, for the whole loop, an angle of $0 \times 4=0$ is obtained, and resonance occurs. Thus, miniaturization would be achieved.

A conventional loop filter, with dimensions $\mathrm{w}_{1}=0.4 \mathrm{~mm}, 1$ $=14 \mathrm{~mm}, \mathrm{~d}_{1}=1.4 \mathrm{~mm}, \mathrm{w}_{\text {port }}=1 \mathrm{~mm}, \mathrm{w}_{\text {feed }}=0.4 \mathrm{~mm}, \mathrm{~g}_{\text {feed }}=$ $0.2 \mathrm{~mm}$, is designed and simulated using CST Microwave Studio, and the results are shown in the inset of Fig. 2. The resonance frequency is $3.24 \mathrm{GHz}$, which is compatible with the predicted result of $3.1 \mathrm{GHz}$ from $\mathrm{S}_{21}$ curve.

Then, all four arms of the filter are loaded by SP metamaterials, and simulation results of insertion loss with varying the width of the SP cells " $b$ " is shown in Fig. 3. It is observed that, due to applying SP resonators, some additional resonances are introduced in the filter response. It is noted that introducing SP cells, with $b=4 \mathrm{~mm}$, also results in considerable bandwidth improvement from 7\% (3.13 GHz$3.27 \mathrm{GHz})$ to $13.4 \%(2.58 \mathrm{GHz}-2.95 \mathrm{GHz})$. It is obvious that by changing parameters of SPs, the frequency of resonances varies, and larger values of SP cells results in more resonance frequency reduction. 


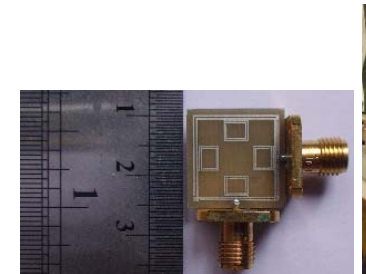

(a)

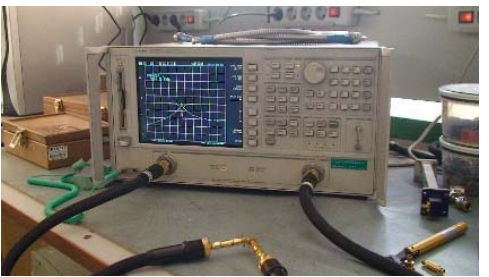

(b)
Fig. 5. (a) The fabricated filter, (b) measurement setup.

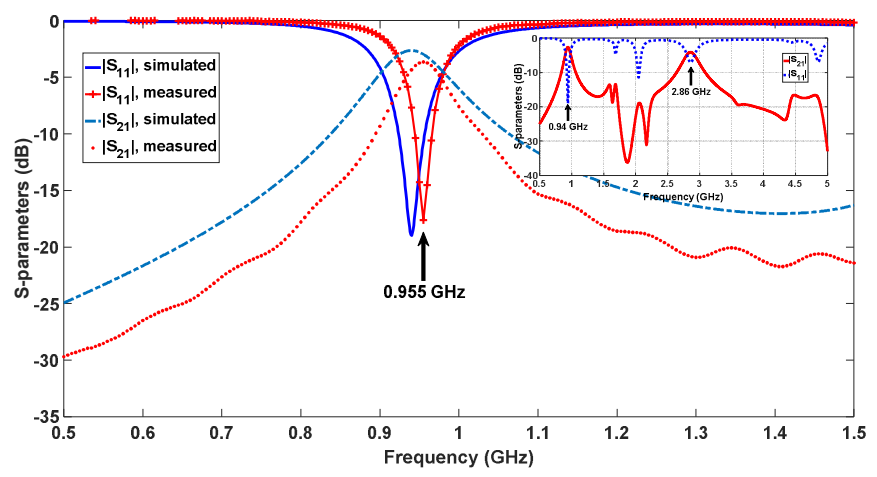

Fig. 6. Simulated and measured S-parameters of the fabricated filter.

TABLE 1

DIMENSIONS OF THE FINAL STRUCTURE

\begin{tabular}{|c|c|c|c|c|c|}
\hline parameter & $\begin{array}{c}\text { Value, } \\
\mathrm{mm}\end{array}$ & parameter & $\begin{array}{c}\text { Value, } \\
\mathrm{mm}\end{array}$ & parameter & $\begin{array}{c}\text { Value, } \\
\mathrm{mm}\end{array}$ \\
\hline $\mathrm{l}$ & 14 & $\mathrm{a}$ & 4 & $\mathrm{w}_{\mathrm{f}}$ & 0.2 \\
\hline $\mathrm{W}_{1}$ & 0.8 & $\mathrm{~b}$ & 3.3 & $\mathrm{~g}_{\mathrm{f}}$ & 0.1 \\
\hline $\mathrm{W}_{\text {line }}$ & 0.2 & $\mathrm{~d}$ & 0.1 & $\mathrm{1}_{\mathrm{fl}}$ & 6 \\
\hline $\mathrm{l}_{\text {line }}$ & 0.1 & $\mathrm{~W}$ & 0.2 & $\mathrm{l}_{\mathrm{f} 2}$ & 6 \\
\hline $\mathrm{W}_{\text {cut }}$ & 0.1 & $\mathrm{~g}$ & 0.2 & $\mathrm{w}_{\text {spu }}$ & 0.2 \\
\hline $\mathrm{W}_{\text {port }}$ & 1 & $\mathrm{w}_{\mathrm{fl}}$ & 0.1 & $\mathrm{1}_{\text {spu }}$ & 13 \\
\hline
\end{tabular}

As mentioned, through the insertion of a gap in the loop resonator frequency reduction would have resulted. Thus, to further compactness of the proposed filter, SP cells are applied to the loop resonator loaded by a gap. Then, to provide stronger coupling to the feed and improving insertion loss, buried feed is applied [14]. Finally, in order to enhance the filter matching, a slot is included in two arms of the filter. The final structure is shown in Fig. 4 and the dimensions are listed in Table 1.

\section{MEASUREMENT RESULTS}

The proposed final filter is fabricated and tested using Agilent 8722ES Network Analyzer in the laboratory of ITRC Research Institute. The filter prototype, as well as the measurement setup, are shown in Fig. 5. Scattering parameters of the fabricated prototype are plotted in Fig. 6 and compared with the simulated ones. As it is illustrated in the figure, measurement results confirm high miniaturization of the proposed design. The small discrepancy between the simulated and measured resonant frequencies is due to fabrication tolerances and the small gap that is created between the connector and the board. Simulated response of the final design is shown in the inset of Fig. 6. Rejection band is relatively wide, and the first undesired resonance occurs at $2.86 \mathrm{GHz}$, which is approximately three times greater than the pass-band resonance frequency.
TABLE 2

COMPARISON WITH RECENT LITERATURE

\begin{tabular}{|c|c|c|c|c|}
\hline $\begin{array}{c}\text { refere } \\
\text { nce }\end{array}$ & year & $\mathrm{f}_{0}, \mathrm{GHz}$ & Size, $\lambda_{g}^{3}$ & $\begin{array}{c}\text { Normalized } \\
\text { size }\left(\lambda_{g}^{3}\right)\end{array}$ \\
\hline$[4]$ & 2005 & 1.45 & $0.29 \times 0.29 \times 0.005$ & 7.53 \\
\hline$[11]$ & 2003 & 1.603 & $0.225 \times 0.225 \times 0.022$ & 19.9 \\
\hline$[13]$ & 2019 & 0.9 & $0.497 \times 0.311 \times 0.01$ & 27 \\
\hline$[15]$ & 2019 & $\mathrm{TX}: 1.95$ & $\mathrm{TX}: 0.205 \times 0.092 \times 0.0078$ & 2.6 \\
& & $\mathrm{RX}: 2.14$ & $\mathrm{RX}: 0.234 \times 0.104 \times 0.0085$ & 3.7 \\
\hline$[16]$ & 2018 & 2.12 & $0.198 \times 0.143 \times 0.0066$ & 3.35 \\
\hline$[17]$ & 2019 & 3.5 & $0.21 \times 0.13 \times 0.0136$ & 6.64 \\
\hline$[18]$ & 2010 & $\mathrm{~A}: 2.4$ & $0.116 \times 0.115 \times 0.006$ & 1.43 \\
& & $\mathrm{~B}: 2.45$ & $0.108 \times 0.116 \times 0.0061$ & 1.37 \\
\hline$[19]$ & 2018 & 6.85 & $0.408 \times 0.332 \times 0.116$ & 281.3 \\
\hline$[20]$ & 2019 & 7.45 & $0.68 \times 0.24 \times 0.029$ & 84.7 \\
\hline$[21]$ & 2015 & 1.8 & $0.19 \times 0.15 \times 0.0089$ & 4.54 \\
\hline$[22]$ & 2017 & 2.45 & $0.168 \times 0.107 \times 0.012$ & 3.86 \\
\hline $\begin{array}{c}\text { This } \\
\text { work }\end{array}$ & & 0.955 & $0.092 \times 0.092 \times 0.0066$ & 1 \\
\hline
\end{tabular}

With considering measured resonant frequency of $0.955 \mathrm{GHz}, \lambda_{g}=\frac{\lambda_{0}}{n}=151.69$ and the fabricated filter occupies a compact volume of $14 \times 14 \times 1 \mathrm{~mm}^{3}=$ $0.045 \times 0.045 \times 0.003 \lambda_{0}^{3}$ or $0.092 \times 0.092 \times 0.0066 \lambda_{g}^{3}$. The fabricated filter is compared with the designs of recently published works in Table 2.

\section{CONCLUSION}

It has analytically been shown that loading microstrip lines by MTM resonators has provided resonant frequency reduction for one wavelength loop resonators. This feature has been used to design and fabricate compact microstrip open loop filters. Spiral MTM cells, due to compactness compared to SRR cells, have been applied. In order to further enhance filter insertion loss and matching, buried feed and an included slot on two of the resonator arms have been applied. The proposed design has been fabricated and tested. Measurement results have confirmed the compactness and effectiveness of the designed filter.

\section{REFERENCES}

J. Carroll and $\mathrm{K}$. Chang, "Microstrip mode suppression ring resonator," Electronics Letters, vol. 30, no. 22, pp. 1861-1862, 1994.

J.-S. Hong and M. J. Lancaster, "Bandpass characteristics of new dual-mode microstrip square loop resonators," Electronics Letters, vol. 31, no. 11, pp. 891-892, 1995.

U. Karacaoglu, D. Sanchez-Hernandez, I. D. Robertson, and M. Guglielmi, "Harmonic suppression in microstrip dual-mode ringresonator bandpass filters," in 1996 IEEE MTT-S International Microwave Symposium Digest, 1996, vol. 3: IEEE, pp. 16351638.

B. T. Tan, J. J. Yu, S. T. Chew, M.-S. Leong, and B.-L. Ooi, "A miniaturized dual-mode ring bandpass filter with a new perturbation," IEEE transactions on microwave theory and techniques, vol. 53, no. 1, pp. 343-348, 2005.

J.-S. Hong and M. J. Lancaster, "Microstrip bandpass filter using degenerate modes of a novel meander loop resonator," IEEE Microwave and guided wave letters, vol. 5, no. 11, pp. 371-372, 1995

L. Zhu and $\mathrm{K}$. Wu, "A joint field/circuit model of line-to-ring coupling structures and its application to the design of microstrip dual-mode filters and ring resonator circuits," IEEE transactions on microwave theory and techniques, vol. 47, no. 10, pp. 19381948, 1999. 
[7] L.-H. Hsieh and K. Chang, "Dual-mode quasi-elliptic-function bandpass filters using ring resonators with enhanced-coupling tuning stubs," IEEE Transactions on Microwave Theory and Techniques, vol. 50, no. 5, pp. 1340-1345, 2002.

[8] M. Matsuo, H. Yabuki, and M. Makimoto, "Dual-mode steppedimpedance ring resonator for bandpass filter applications," IEEE Transactions on Microwave Theory and Techniques, vol. 49, no. 7, pp. 1235-1240, 2001.

[9] A. C. Kundu and I. Awai, "Control of attenuation pole frequency of a dual-mode microstrip ring resonator bandpass filter," IEEE Transactions on Microwave Theory and Techniques, vol. 49, no. 6, pp. 1113-1117, 2001.

[10] A. Gorur, "A novel dual-mode bandpass filter with wide stopband using the properties of microstrip open-loop resonator," IEEE Microwave and Wireless components letters, vol. 12, no. 10, pp. 386-388, 2002.

[11] A. Gorur, C. Karpuz, and M. Akpinar, "A reduced-size dualmode bandpass filter with capacitively loaded open-loop arms," IEEE microwave and wireless components letters, vol. 13, no. 9, pp. 385-387, 2003.

[12] J.-S. Hong and M. J. Lancaster, "Theory and experiment of novel microstrip slow-wave open-loop resonator filters," IEEE Transactions on Microwave theory and Techniques, vol. 45, no. 12, pp. 2358-2365, 1997.

[13] A. Harikrishnan, S. Mridula, and P. Mohanan, "Stub loaded hexagonal open loop band pass filter with improved selectivity," in 2019 URSI Asia-Pacific Radio Science Conference (AP$R A S C)$, 2019: IEEE, pp. 1-4.

[14] A. Naji and P. A. Warr, "Geometrical perturbation techniques and Approximate Analysis for eigenmode splitting and shifting in electromagnetic planar Dual-Mode Resonators," Scientific reports, vol. 9, no. 1, pp. 1-14, 2019.

[15] A. Elzayat and A. Kouki, "Miniaturized planar filters exhibiting flexible placement of three transmission zeros suitable for duplexer design," International Journal of RF and Microwave Computer Aided Engineering, vol. 29, no. 1, p. e21501, 2019.

[16] A. Ghaderi, A. Golestanifar, and F. Shama, "Microstrip bandpass filters using coupled feed lines for third and fourth generation communications," AEU-International Journal of Electronics and Communications, vol. 86, pp. 195-201, 2018.

[17] G. Karimi, Y. Pourasad, A. Lalbakhsh, H. Siahkamari, and B. Mohamadzade, "Deign of a compact ultra-narrow band dual band filter for WiMAX application," AEU-International Journal of Electronics and Communications, vol. 110, p. 152827, 2019.

[18] X. Luo, H. Qian, J.-G. Ma, K. Ma, and K. S. Yeo, "Compact dualband bandpass filters using novel embedded spiral resonator (ESR)," IEEE microwave and wireless components letters, vol. 20, no. 8, pp. 435-437, 2010.

[19] M. G. Mayani, S. Asadi, A. Pirhadi, and S. M. Mahani, "Design and analysis of a super compact wide-band bandpass filter based on metamaterial resonators," AEU-International Journal of Electronics and Communications, vol. 97, pp. 79-84, 2018.

[20] M. Riaz, B. S. Virdee, P. Shukla, and M. Onadim, "Quasi-elliptic ultra-wideband bandpass filter with super-wide stopband," $A E U$ International Journal of Electronics and Communications, vol. 105, pp. 171-176, 2019.

[21] Y. Zhang, L. Gao, and X. Y. Zhang, "Compact quad-band bandpass filter for DCS/WLAN/WiMAX/5G Wi-Fi application," IEEE Microwave and Wireless Components Letters, vol. 25, no. 10, pp. 645-647, 2015.

[22] Y. Zhang and X.-L. Zhao, "Compact tri-band bandpass filter with two closely frequency spaced passbands," Journal of ElEctromagnEtic WavEs and applications, vol. 31, no. 1, pp. 8290, 2017.

[23] M. Alibakhshikenari, B. S. Virdee, M. Khalily, P. Shukla, C. H. See, R. Abd-Alhameed, F. Falcone, and E. Limiti, "Beamscanning leaky-wave antenna based on CRLH-metamaterial for millimetre-wave applications." IET Microwaves, Antennas \& Propagation, vol. 13, no. 8, pp. 1129-1133, 2019.

[24] S. P. Khanjari, S. Jarchi, M. Mohammad-Taheri, "Compact and wideband planar loop antenna with microstrip to parallel strip balun feed using metamaterials," AEU-International Journal of Electronics and Communications, vol. 111, p. 152883, 2019.

[25] F. Bilotti, A. Toscano, L. Vegni, K. Aydin, K. B. Alici, and E. Ozbay, "Equivalent-circuit models for the design of metamaterials based on artificial magnetic inclusions," IEEE Transactions on Microwave Theory and Techniques, vol. 55, no. 12, pp. 2865-2873, 2007.

[26] F. Bilotti, A. Toscano, and L. Vegni, "Design of spiral and multiple split-ring resonators for the realization of miniaturized metamaterial samples," IEEE transactions on antennas and propagation, vol. 55, no. 8, pp. 2258-2267, 2007. 